\title{
Estrogen and estrogen receptor induce matrix metalloproteinase-26 expression in endometrial carcinoma cells
}

\author{
HIROTAKA NISHI $^{1}$, MASAHIKO KURODA ${ }^{2}$ and KEIICHI ISAKA ${ }^{1}$ \\ Departments of ${ }^{1}$ Obstetrics and Gynecology, and ${ }^{2}$ Molecular Pathology, \\ Tokyo Medical University, Shinjuku-ku, Tokyo 160-0023, Japan
}

Received March 29, 2013; Accepted May 3, 2013

DOI: $10.3892 /$ or.2013.2527

\begin{abstract}
The human matrix metalloproteinase (MMP)-26, also called matrilysin- 2 or endometase, has been isolated as a matrilysin (MMP-7) homolog. Several reports describe that MMP-26 may be related to the development of endometrial carcinomas. Total RNAs were isolated from 51 normal endometrial tissue samples, 6 endometrial hyperplasia tissue samples and 30 endometrial carcinomas. Real-time reverse transcription-polymerase chain reaction (RT-PCR) was performed to evaluate MMP-26 mRNA expression levels. We examined the effect of estrogen and its receptor (ER) on MMP-26 expression in endometrial carcinoma cell lines by real-time RT-PCR, western blot analysis and luciferase assays. To examine protein-DNA binding between ER and MMP-26 promoter, we performed chromatin immunoprecipitation (ChIP) assay. Real-time RT-PCR analysis revealed that MMP-26 mRNA expression was significantly higher in the normal human endometria and hyperplasias compared with that in endometrial carcinomas. Estrogen not only transactivated the MMP-26 promoter activity but also enhanced endogenous MMP-26 expression. The MMP-26 promoter region contains a putative ER response element (ERE). Nuclear ER protein interacted with ERE on the MMP-26 promoter by ChIP assay. We found a significant difference in MMP-26 expression in normal and malignant endometrial tissue samples and that estrogen induced MMP-26 expression. Estrogen may induce endometrial hyperplasia but not endometrial carcinoma. Our results provide evidence that regulation of MMP-26 promoter activity by estrogen may represent a mechanism for endometrial carcinogenesis.
\end{abstract}

Correspondence to: Dr Hirotaka Nishi, Department of Obstetrics and Gynecology, Tokyo Medical University, 6-7-1 Nishishinjuku, Shinjuku-ku, Tokyo 160-0023, Japan

E-mail: nishih@tokyo-med.ac.jp

Key words: estrogen receptor, MMP-26, matrilysin-2, endometase, endometrium, transcription

\section{Introduction}

Matrix metalloproteinases (MMPs) are a family of zincdependent endopeptidases that degrade the extracellular matrix. These enzymes are produced by many cell types in response to inflammation or tumor invasion. Among the variety of proteinases potentially implicated in tumor invasion, the MMPs have attracted considerable interest because of their ability to collectively degrade essentially all protein constituents of connective tissues (1). Substantial evidence indicates that the process of the degradation of extracellular matrix and basement membranes is largely mediated by the combined action of proteolytic enzymes, thus, facilitating tumor invasion and metastasis (2). To date, 30 different human MMPs have been cloned and characterized at the amino acid sequence level (3). The correlation between MMPs and malignant tumors is well documented both in vitro and in vivo (4). In general, MMPs levels are abnormally induced in metastatic tumor cells and are associated with invasive lesions and poor clinical prognosis in cancer patients $(5,6)$. However, new findings of the MMP family and their subsequent functional characterization at both genetic and biochemical levels indicate new views on the role of these enzymes in tumor progression (7). Evidence is accumulating that MMPs are not exclusively involved in the proteolysis of the tissue barriers for metastatic spread. Actually, MMPs have been reported to play direct roles in other critical events for tumor evolution that occur at earlier stages in carcinogenesis, events such as tumor promotion, modulation of the growth of the primary tumor and angiogenesis (8).

Recently, the human matrix metalloproteinase (MMP)-26, also called matrilysin-2 or endometase, has been isolated as a matrilysin (MMP-7) homolog (3,9-13). Analysis of the proteolytic specificity showed that MMP-26 is able to degrade a wide range of proteins present in the extracellular matrix and basement membrane such as fibronectin, fibrinogen, vitronectin, $\alpha 1$-antitrypsin, $\alpha 2$-macroglobulin and denatured collagen (types I-IV). While the biological function of MMP-26 is not yet fully understood, several reports describe that MMP-26 may be related to the development of endometrial carcinoma $(13,14)$. In the present study, we examine the MMP-26 expression level in normal and malignant endometrial tissue samples. 
As estrogen is a potent inducer of endometrial proliferation in vivo, it may play a role in the regulation of MMP-26. It is believed that estrogen is related to the development of endometrial hyperplasia which is the precursor of endometrial carcinoma $(15,16)$. Li et al $(17)$ described that estrogen-estrogen receptor (ER) complex activated the MMP-26 expression. However, the molecular mechanisms by which ER activates MMP-26 have not been examined in any detail. Interestingly, computer-assisted homology searches have revealed potential binding sites for ER in the MMP-26 proximal promoter. Therefore, we also examine the correlation between estrogen-ER complex and MMP-26 expression.

\section{Materials and methods}

Samples. The samples were obtained with informed consent from patients undergoing surgery or biopsy at Tokyo Medical University Hospital. We used 51 normal endometria, 6 endometrial hyperplasias and 30 endometrial carcinomas. The tissues were finely minced into small pieces with scissors, washed in phosphate-buffered saline (PBS), snap-frozen and stored at $-80^{\circ} \mathrm{C}$ until use for RNA extraction.

Cell cultures and estrogen treatment. The examined cell lines. $\mathrm{EN}$ and Ishikawa, were derived from endometrial carcinoma. EN does not express ER $\alpha$ (18). Ishikawa which expressed ER $\alpha$ was kindly provided by Dr Masato Nishida (Department of Obstetrics and Gynecology, National Kasumigaura Hospital, Tsuchiura, Japan) (19). These cells were grown in DMEM supplemented with $10 \%$ fetal bovine serum, $100 \mathrm{U} / \mathrm{ml}$ penicillin G sodium and $100 \mu \mathrm{g} / \mathrm{ml}$ streptomycin sulfate in the presence of $5 \% \mathrm{CO}_{2}$ at $37^{\circ} \mathrm{C}$.

The cells were plated at a density of $2.5 \times 10^{4}$ cells $/$ well in 24-well plates and allowed to attach overnight. After serum starvation, these cells were treated with vehicle, $17 \beta$-estradiol $\left(\mathrm{E}_{2} ; 1-10 \mathrm{nM}\right)$ or $\mathrm{E}_{2}(1-10 \mathrm{nM})+$ ICI $182780(1-10 \mathrm{mM})$ with $2 \%$ charcoal-stripped serum. $\mathrm{E}_{2}$ and ICI 182780 were obtained from Sigma Chemical Co. (St. Louis, MO, USA).

Real-time RT-PCR analysis. Total RNA was isolated using Isogen reagent (Nippon Gene, Tokyo, Japan) and quantified by A260/A280 measurement using an Ultrospec 3000 (Amersham Pharmacia Biotech). Total RNA (5 $\mu \mathrm{g})$ was reverse transcribed into cDNAs. Real-time PCR was performed for the quantitative estimation. PCR reactions ( $20 \mu 1)$ were set up with final concentrations of $5 \mathrm{~mm} \mathrm{MgCl}, 2 \mu \mathrm{l}$ SYBR-Green Master Mix (Roche Molecular Biochemicals), $5 \mu 1$ 1:10 diluted cDNA, and $0.3 \mu \mathrm{M}$ of both forward and reverse primers which are as follows: 5'-CATCCGCAGTGAAAGACAGTA-3' (MMP-26-F) 5'-TTGCAACCAGGAACAGATTAT-3' (MMP-26-R).

The reactions were then cycled in the LightCycler (Roche Molecular Biochemicals) with the following parameters: denaturation for 1 cycle at $95^{\circ} \mathrm{C}$ for $10 \mathrm{sec}, 45$ cycles (temperature transition of $20^{\circ} \mathrm{C} / \mathrm{sec}$ ) of $95^{\circ} \mathrm{C}$ for $0 \mathrm{sec}, 50^{\circ} \mathrm{C}$ for $10 \mathrm{sec}$, and $72^{\circ} \mathrm{C}$ for $15 \mathrm{sec}$ and fluorescence reading taken at $72^{\circ} \mathrm{C}$, melting curve analysis with continuous fluorescence reading. If necessary, optimization of the protocol was achieved by changing $\mathrm{MgCl}_{2}$ concentrations and/or reading fluorescence at a higher temperature and/or using LightCycler FastStart DNA
Master SYBR-Green I (Roche Molecular Biochemicals). The LightCycler software generated a standard curve (measurements taken during the exponential phase of the amplification) that enabled the amount of each gene in each test sample to be determined. The amount of each gene was normalized by that of the GAPDH.

Immunohistological detection. Anti-human MMP-26 peptide polyclonal antibodies were developed in rabbits as described (14). Immunochistochemical saining was carried out using Histofine SAB-PO (R) kit (Nichirei Corp., Tokyo, Japan). Paraffin sections were de-paraffinized and blocked with methanol containing $3 \% \mathrm{H}_{2} \mathrm{O}_{2}$, sequentially $10 \%$ goat normal serum. Sections were incubated with a rabbit anti-MMP-26 (1:1,000 dilution) for $1 \mathrm{~h}$ at room temperature. Sections were then sequentially treated with a biotinylated goat anti-rabbit antibody and a horseradish peroxidase (HRP) labeled streptoavidin. Development was done by treating the sections with a Liquid DAB-Plus Substrate kit (Zymed Laboratories Inc., San Francisco, CA, USA). After counterstaining with hematoxylin, immunostaining of MMP-26 on the tissue sections was detected by light microscope.

Western blot analysis. Cells and tissues were lysed on ice for $30 \mathrm{~min}$ in lysis buffer [10 mM Tris at $\mathrm{pH} 8.0,1 \mathrm{mM}$ EDTA, $400 \mathrm{mM} \mathrm{NaCl}, 10 \%$ glycerol, $0.5 \%$ NP-40, $5 \mathrm{mM}$ sodium fluoride, $0.1 \mathrm{mM}$ phenylmethylsulfonyl fluoride, $1 \mathrm{mM}$ DTT], containing complete protease inhibitor cocktail (Boehringer Mannheim, Indianapolis, IN, USA). The lysate was subjected to centrifugation at $14,000 \mathrm{rpm}$ for $15 \mathrm{~min}$ and the soluble fraction collected. Protein concentrations were measured using a Bio-Rad protein assay kit (Bio-Rad Laboratories, Hercules, CA, USA). Equal amounts of protein $(40 \mu \mathrm{g})$ were loaded onto a 4-12\% SDS-polyacrylamide gel and subjected to electrophoresis at $200 \mathrm{~V}$ for $50 \mathrm{~min}$. The protein was transferred onto a PVDF membrane and probed with anti-MMP-26 antibody (14) and anti-actin antibody (C4) (Boehringer Mannheim). The same blot was probed after stripping the membrane with the different antibodies. Each protein was detected by horseradish peroxidase-conjugated secondary antibody coupled with enhanced chemiluminescence (ECL) western blotting detection reagents (Amersham Pharmacia Biotech, Sweden Uppsala). Each western blot analysis was performed at least twice. Each band intensity was normalized by the intensity of the actin band.

DNA plasmids. The ER expression vectors were previously described (20). pMMP-26-luc is an MMP-26 promoter-luciferase reporter plasmid. A 1000-bp sequence upstream of the translation start site of MMP-26 is cloned into pGL3-Basic (Promega) at HindIII sites. PCR was performed to amplify this MMP-26 promoter region using both forward and reverse primers which are as follows: MMP-26/clon1-U5'-CCCAAG CTTGACAAATGAGGGTTTGGCAT-3' MMP-26/clon804L5'-CCGCTCGAGTTAAGGTATGTCAGATGAAC-3'.

Transfections and luciferase assays. Cells were seeded at $2 \times 10^{4}$ cells/well in 96-well plate and incubated overnight at $37^{\circ} \mathrm{C}$ in a $5 \% \mathrm{CO}_{2}$ incubator. For each transfection, $10 \mathrm{ng}$ of empty vector and/or expression vectors along with 
1-100 ng of promoter-luciferase DNA were mixed in $25 \mu 1$ of Opti-MEM (Life Technologies) and a precipitate formed using Lipofectamine 2000 (Life Technologies) according to the manufacturer's recommendations. Cells were washed with Opti-MEM and complexes were applied to the cells with or without 1-100 nM E $\mathrm{E}_{2}$. Twenty-four hours after transfection, cells were harvested and extracts were prepared with the Glo Lysis Buffer (Promega, Madison, WI, USA). Luciferase activity was measured in extracts from triplicate samples using the Bright-Glo Luciferase Assay system (Promega).

Chromatin immunoprecipitation assay. Chromatin immunoprecipitation assays were performed using the ChIP Assay kit (Upstate Biotechnology Inc., Lake Placid, NY, USA) according to the manufacturer's protocol. EN cells incubated with/without overexpression of ER in 10-cm dishes for $24 \mathrm{~h}$ were cross-linked by treatment with formaldehyde (final concentration, 1\%) for $10 \mathrm{~min}$ at room temperature. After washing with PBS, cells were pelleted down and resuspended in SDS lysis buffer [1\% SDS, $10 \mathrm{mM}$ EDTA, $50 \mathrm{mM}$ Tris-HCI (pH 8.1), $1 \mathrm{mM}$ DTT, $1 \mathrm{mM}$ PMSF). The lysates were then subjected to sonication to break DNA to the length of $500-1,000 \mathrm{bp}$, diluted with dilution buffer $[0.01 \%$ SDS, 1.1\% Triton X-100, 1.2 mM EDTA, 16.7 mM Tris-HCI ( $\mathrm{pH} 8.1), 167 \mathrm{mM} \mathrm{NaCl}]$ and pre-cleared by incubating with Salmon Sperm DNA/protein A agarose 50\% slurry for $60 \mathrm{~min}$ at $4^{\circ} \mathrm{C}$. The supernatant was incubated with anti-ER $\alpha$ (sc-786X, Santa Cruz Biotechnology) antibody at $4^{\circ} \mathrm{C}$ overnight. Immunocomplex was collected with Salmon Sperm DNA/protein A Agarose 50\% slurry and eluted after extensive washings, and cross-linkage was reversed by heating at $65^{\circ} \mathrm{C}$, followed by treatment with $40 \mathrm{mg} / \mathrm{ml}$ proteinase $\mathrm{K}$ at $45^{\circ} \mathrm{C}$ for $60 \mathrm{~min}$. DNA was recovered by phenol-chloroform/ethanol precipitation, and was used as a template for nested PCR to amplify the target sites in the MMP-26 promoter. The target sites and the corresponding primer sequences are shown in Fig. 4A. The PCR products were electrophoresed on a $1.0 \%$ agarose gel and visualized by ethidium bromide staining.

\section{Results}

MMP-26 expression is downregulated in endometrial carcinomas. The expression of MMP-26 mRNA was examined in 30 endometrial carcinoma, 6 endometrial hyperplasia patients and normal endometrium from 51 patients who suffered from uterine myoma or prolapse of the uterus. Quantitative real-time RT-PCR analysis revealed that normal endometria expressed significantly higher levels of MMP-26 mRNA than the endometrial carcinomas tissues (Fig. 1A). However, endometrial hyperplasias showed the similar level of MMP-26 as normal endometria (Fig. 1A).

Furthermore, we examined MMP-26 expression in protein level by immunohistochemical analysis using anti-MMP-26 antibodies. MMP-26 expression was observed only in epithelial glandular cells but not in stromal cells (Fig. 1B). No stained or faintly stained carcinoma cells were observed in endometrial carcinoma tissues (Fig. 1C).

Estrogen induces the endogenous MMP-26 expression. We examined the effect of estrogen on MMP-26 protein expres-
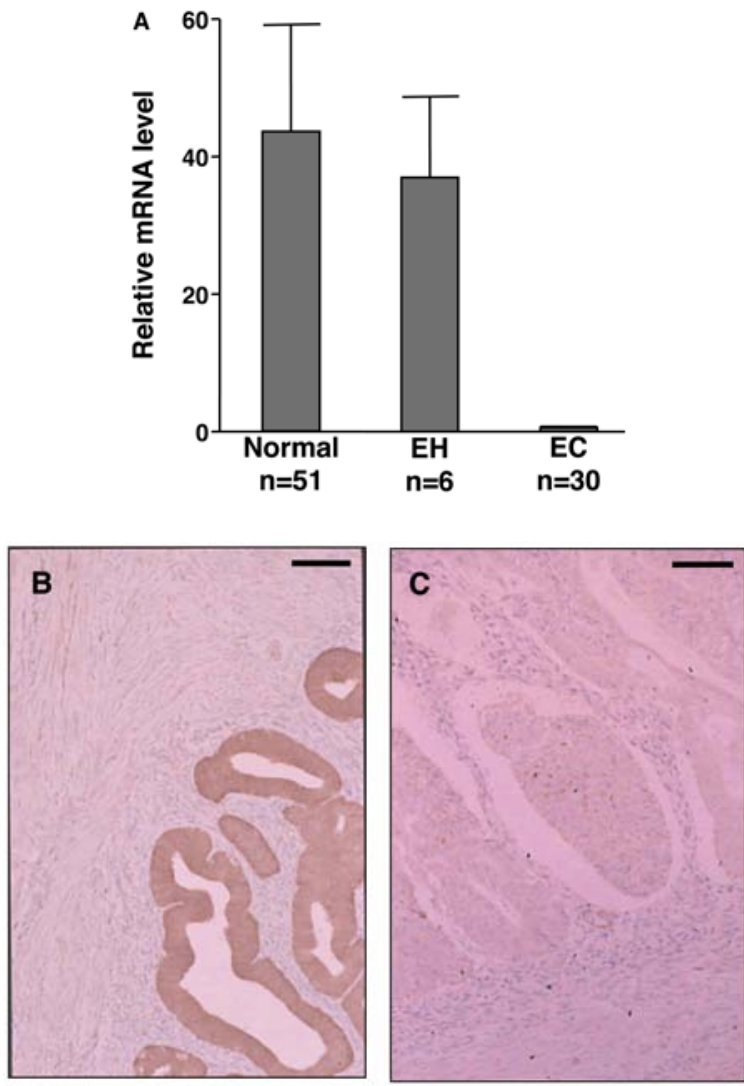

Figure 1. (A) MMP-26 mRNA level in normal endometrial, endometrial hyperplasias $(\mathrm{EH})$ and endometrial carcinomas (EC). (B and C) Immunohistochemical localization of MMP-26. (B) Normal endometrium (original magnification, $\mathrm{x} 100)$. (C) Endometrial cancer (original magnification, $\mathrm{x} 100$ ). Bar, $100 \mu \mathrm{m}$.
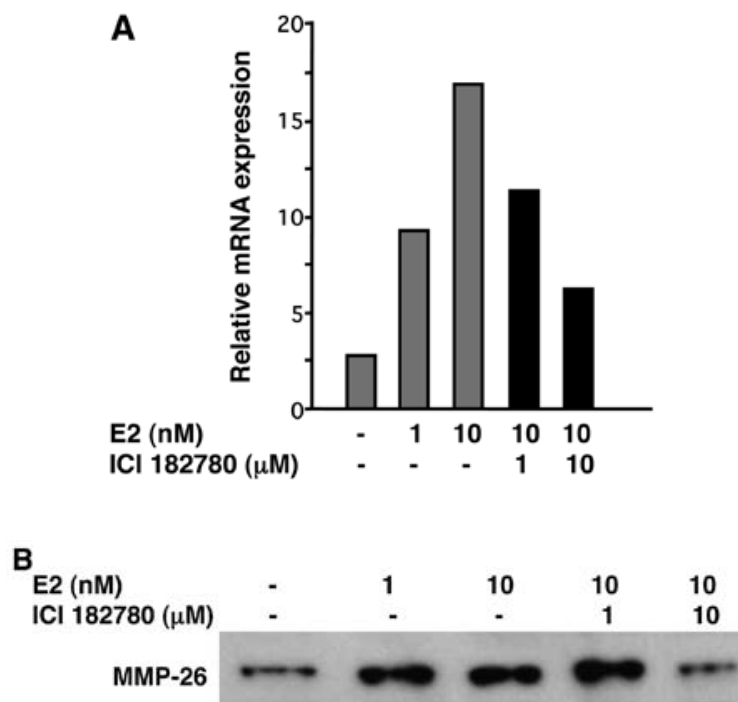

Actin

Figure 2. (A) Estrogen induces MMP-26 mRNA expression in Ishikawa. (B) Estrogen induces MMP-26 protein expression in Ishikawa.

sion in ER $\alpha$ positive endometrial cancer cell line, Ishikawa. $\mathrm{E}_{2}$ at 1-10 nM dose-dependently increased both MMP-26 mRNA and protein expression levels in Ishikawa cells (Fig. 2). 

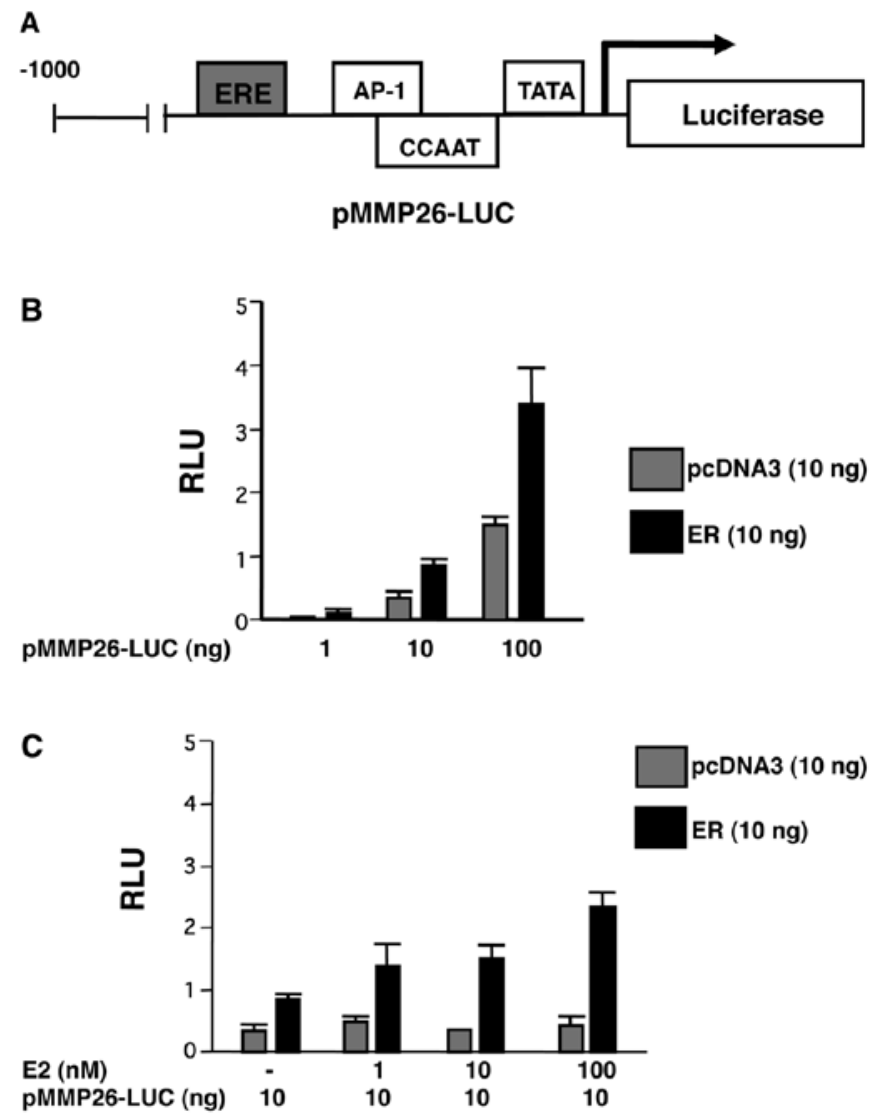

Figure 3. (A) Schema of the pMMP-26-luc. (B) ER Overexpression transactivates the MMP-26 promoter in EN. (C) Estrogen transactivates the MMP-26 promoter in EN.

The upregulated expression of MMP-26 protein was blocked by pretreatment of cells with the estrogen receptor inhibitor, ICI 182780, dose-dependently, indicating that the estrogen receptor was involved (Fig. 2).

Estrogen and ER transactivate the MMP-26 promoter. It is probably likely that MMP-26 is regulated by estrogen through an estrogen-response element (ERE). Bioinformatics in silico analysis was performed for extensive evaluation of the MMP-26 gene promoter sequence. We looked for the presence of ERE like motifs (GGTCANNNTGACC) in the MMP-26 gene and found it in the promoter region of the MMP-26 gene between nucleotides -129 and -117 (5'-GGTCACTCTTGCCC-3'). This has the characteristic of the ERE, a 13-bp palindromic element consisting of two 5-bp arms separated by a 3-bp spacer. This ERE motif in the MMP-26 promoter has one arm of the perfect palindromic element sequences and a second incomplete set. These two arms of the palindrome are separated by the 3-bp spacing which is essential for ER binding. We show that the relative positions of the ERE and other binding sites for transcription factors in the MMP-26 promoter in Fig. 3A.

To examine whether induction of MMP-26 gene is regulated directly by estrogen through ligand-dependent transcriptional factors that bind DNA at conserved ERE, EN cells were transfected with pMMP-26-luc for luciferase assays. ER overexpression activated the MMP-26 promoter

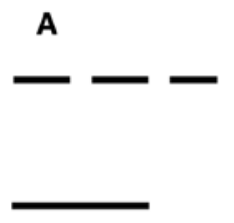

MMP67: 5'- ACGTGTGCGTGTCAACTTAT -3' MMP356: 5'- ATTAGCCGGGTGTGGTGGTG -3' (1st PCR) MMP73: 5'- GCGTGTCAACTTATTATCAA - $3^{\prime}$ MMP356: 5'- ATTAGCCGGGTGTGGTGGTG -3' (2nd PCR) MMP669: 5'- ССTCCTGTTTCTTGTTTTCC -3' MMP889: 5'- CTCTGCCAGTTTGCCCTTTC -3' (1st PCR) MMP725: 5'- GACTGACCTGTATTTCCTTA - $3^{\prime}$ MMP884: 5'- CCAGTTTGCCCTTTCTTTGA -3' (2nd PCR)

OXY77: 5'- TATTGGGAGGCTGGATAAAG -3' OXY332: 5'- TGGGCATTGCGTCGGTGGAG -3' (1st PCR) OXY80: 5'- TGGGAGGCTGGATAAAGTCT -3' OXY220: 5'- CTGGGCCGGGGTCAAGGTCA -3' (2nd PCR)
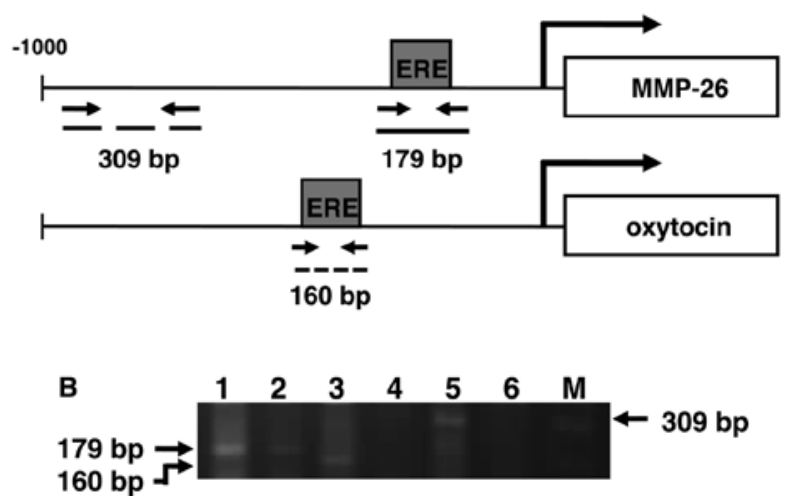

Figure 4. (A) Primer sequences targeting various regions on the MMP-26 and oxytocin promoter used for ChIP assays and schema of each promoter region. (B) ER interacts with MMP-26 promoter. Lane 1, ERE/MMP-26 input; lane 2, ERE/MMP-26; lane 3, ERE/oxytocin; lane 4, ERE/MMP-26/ER (-); lane 5, MMP-26 promoter input; lane 6, MMP-26 promoter; lane M, marker.

2-fold (Fig. 3B). Further investigation showed that 1-100 nM $\mathrm{E}_{2}$ significantly enhanced the luciferase activity by $2.5-5$-fold (Fig. 3C). These results suggest that there may be a functional ERE motif that mediates estrogen-dependent MMP-26 expression in the promoter fragment of MMP-26.

ER interacts with the putative ERE in the MMP-26 promoter. To confirm the existence of an ERE in the MMP-26 promoter, we performed in silico analysis and conventional ChIP assays. ChIP analysis demonstrated that only fragments containing the putative ERE were precipitated after administration of ER transfection (Fig. 4B). No PCR product was detected from samples precipitated with $\operatorname{IgG}$ (data not shown). These results verify the ERE in the MMP-26 promoter and indicate that the ER/ERE complex mediates the regulation of MMP-26 expression by estrogen.

\section{Discussion}

It is believed that partly estrogen is related to the development of endometrial carcinoma (21). Actually, estrogen is essential to develop endometrial hyperplasia, whereas other genes, such as PTEN, K-ras, hMLH1 and p53 are critical in endometrial carcinogenesis (22-25). It seems that estrogen is not directly related to the development of endometrial carcinoma. Our data suggest estrogen-dependence of MMP-26 expression and demonstrate the specific association of MMP-26 with endometrial carcinomas. MMP-26 expression was lost in endometrial carcinoma, whereas normal endometrium as well as endometrial hyperplasia has high MMP-26 expression. While the biological function of MMP-26 is not yet fully understood, 
if MMP-26 is essential to development of endometrial carcinoma, estrogen induces endometrial hyperplasia but possibly not endometrial carcinoma.

Li et al (17) described that ER activated the MMP-26 expression. However, the molecular mechanisms by which ER activates MMP-26 have not been examined in any detail. Interestingly, computer-assisted homology searches have revealed potential binding sites for ER in the MMP-26 proximal promoter. We performed luciferase assays and ChIP assays to demonstrate the correlation between ER and MMP-26. The data demonstrate that the ER transactivates the MMP-26 promoter and induces MMP-26 expression.

As in a previous study, to define the physiological role of MMP-26, we have examined the expression pattern of this enzyme in human endometrial tissues. In marked contrast to the restricted expression pattern of this enzyme in endometrial carcinoma, this gene is expressed in normal endometrium and endometrial hyperplasia. The sensitivity and specificity of endometrial carcinoma by cytological analysis is $\sim 70-80 \%$, much lower than those of cervical carcinoma $(26,27)$. In the present study, we show that there is a significant difference in MMP-26 expression between normal endometria and endometrial carcinomas. Combination of cytological analysis and detection of MMP-26 expression might be a useful examination to identify endometrial carcinoma. The detectability of combination examination should be better than that of cytological analysis only. It is also important to measure the expression level of MMP-26 mRNA or protein. The expression level might indicate the malignancy. Additional studies will be necessary to clarify the clinical significance of the MMP-26 expression in human endometrial carcinoma $(4,6)$.

Since endometrial hyperplasia tissues, a precancerous lesion of endometrial carcinomas, did exhibit MMP-26, downregulation of MMP-26 expression might be a critical event in endometrial carcinogenesis. MMP-26 expression may affect the development of endometrial carcinomas. At present, the mechanisms of MMP-26 downregulation and correlation with other genes are not clear. Although our data in the present study are insufficient to make a conclusion, the preliminary data suggest that the MMP-26 gene might play an essential role as a tumor suppressor gene. Additional studies to explain MMP-26 functions, regulation mechanisms, and correlation with other genes, are necessary to fully understand the roles of MMP-26 in tumor biology.

The mechanisms documented in the present study suggest the involvement of MMP-26 in malignant progression of endometrial carcinoma and brings us several steps closer to understanding the functional role of the unconventional MMP-26 enzyme in physiologic and pathologic processes. Further analysis of MMP-26 regulation in endometrial cells could provide further insight into the molecular mechanism and the role of MMP-26 expression or activity in human endometrial carcinogenesis.

\section{Acknowledgements}

Ishikawa was kindly provided by Dr Masato Nishida (Department of Obstetrics and Gynecology, National Kasumigaura Hospital). The present study was supported by JSPS KAKENHI (grant no. 16790973). The authors thank Ms. Chinatsu Higuma (Department of Obstetrics and Gynecology, Tokyo Medical University) for invaluable experimental assistance.

\section{References}

1. Nagase H and Woessner JF Jr: Matrix metalloproteinases. J Biol Chem 274: 21491-21494, 1999.

2. Pilka R, Norata GD, Domanski H, Andersson C, Hansson S, Eriksson P and Casslen B: Matrix metalloproteinase-26 (matrilysin-2) expression is high in endometrial hyperplasia and decreases with loss of histological differentiation in endometrial cancer. Gynecol Oncol 94: 661-670, 2004.

3. Park HI, Ni J, Gerkema FE, Liu D, Belozerov VE and Sang QX: Identification and characterization of human endometase (Matrix metalloproteinase-26) from endometrial tumor. J Biol Chem 275: 20540-20544, 2000.

4. Stetler-Stevenson WG, Aznavoorian S and Liotta LA: Tumor cell interactions with the extracellular matrix during invasion and metastasis. Annu Rev Cell Biol 9: 541-573, 1993.

5. Murray GI, Duncan ME, O'Neil P, Melvin WT and Fothergill JE: Matrix metalloproteinase-1 is associated with poor prognosis in colorectal cancer. Nat Med 2: 461-462, 1996.

6. Nelson AR, Fingleton B, Rothenberg ML and Matrisian LM: Matrix metalloproteinases: biologic activity and clinical implications. J Clin Oncol 18: 1135-1149, 2000.

7. Chambers AF and Matrisian LM: Changing views of the role of matrix metalloproteinases in metastasis. J Natl Cancer Inst 89: 1260-1270, 1997.

8. Sternlicht MD, Lochter A, Sympson CJ, et al: The stromal proteinase MMP3/stromelysin-1 promotes mammary carcinogenesis. Cell 98: 137-146, 1999.

9. Velasco G, Pendas AM, Fueyo A, Knauper V, Murphy G and Lopez-Otin C: Cloning and characterization of human MMP-23, a new matrix metalloproteinase predominantly expressed in reproductive tissues and lacking conserved domains in other family members. J Biol Chem 274: 4570-4576, 1999.

10. de Coignac AB, Elson G, Delneste Y, et al: Cloning of MMP-26. A novel matrilysin-like proteinase. Eur J Biochem 267: 3323-3329, 2000.

11. Marchenko GN, Ratnikov BI, Rozanov DV, Godzik A, Deryugina EI and Strongin AY: Characterization of matrix metalloproteinase-26, a novel metalloproteinase widely expressed in cancer cells of epithelial origin. Biochem J 356: 705-718, 2001.

12. Uria JA and Lopez-Otin C: Matrilysin-2, a new matrix metalloproteinase expressed in human tumors and showing the minimal domain organization required for secretion, latency, and activity. Cancer Res 60: 4745-4751, 2000.

13. TrabertB, Wentzensen N, Yang HP, Sherman ME, Hollenbeck AR, Park Y and Brinton LA: Is estrogen plus progestin menopausal hormone therapy safe with respect to endometrial cancer risk? Int J Cancer 132: 417-426, 2013.

14. Isaka K, Nishi H, Nakai H, Nakada T, Feng Li Y, Ebihara Y and Takayama M: Matrix metalloproteinase-26 is expressed in human endometrium but not in endometrial carcinoma. Cancer 97: 79-89, 2003.

15. Cooke PS, Buchanan DL, Young P, et al: Stromal estrogen receptors mediate mitogenic effects of estradiol on uterine epithelium. Proc Natl Acad Sci USA 94: 6535-6540, 1997.

16. Pierro E, Minici F, Alesiani O, et al: Stromal-epithelial interactions modulate estrogen responsiveness in normal human endometrium. Biol Reprod 64: 831-838, 2001.

17. Li W, Savinov AY, Rozanov DV, et al: Title: Matrix metalloproteinase-26 is associated with estrogen-dependent malignancies and targets $\alpha 1$-antitrypsin serpin. Cancer Res $64: 8657-8665$, 2004.

18. Isaka K, Nishi H, Sagawa Y, et al: Establishment of a new human cell line (EN) with TP53 mutation derived from endometrial carcinoma. Cancer Genet Cytogenet 141: 20-25, 2003.

19. Nishida M: The Ishikawa cells from birth to the present. Hum Cell 15: 104-117, 2002.

20. Mak HY and Parker MG: Use of suppressor mutants to probe the function of estrogen receptor-p160 coactivator interactions. Mol Cell Biol 21: 4379-4390, 2001.

21. Banno K, Kisu I, Yanokura M, et al: Progestin therapy for endometrial cancer: the potential of fourth-generation progestin (Review). Int J Oncol 40: 1755-1762, 2012. 
22. Xiong Y, Dowdy SC, Eberhardt NL, Podratz KC and Jiang SW: hMLH1 promoter methylation and silencing in primary endometrial cancers are associated with specific alterations in MBDs occupancy and histone modifications. Gynecol Oncol 103: 321-328, 2006

23. Tashiro H, Blazes MS, Wu R, et al: Mutations in PTEN are frequent in endometrial carcinoma but rare in other common gynecological malignancies. Cancer Res 57: 3935-3940, 1997.

24. Lax SF, Kendall B, Tashiro H, Slebos RJ and Hedrick L: The frequency of $\mathrm{p} 53$, K-ras mutations, and microsatellite instability differs in uterine endometrioid and serous carcinoma: evidence of distinct molecular genetic pathways. Cancer 88: 814-824, 2000.
25. Kawaguchi M, Banno K, Yanokura M, et al: Analysis of candidate target genes for mononucleotide repeat mutation in microsatellite instability-high (MSI-H) endometrial cancer. Int J Oncol 35: 977-982, 2009.

26. Suprun HZ, Taendler-Stolero R, Schwartz J and Ettinger M: Experience with Endopap endometrial sampling in the cytodiagnosis of endometrial carcinoma and its precursor lesions. I. A correlative cytologic-histologic-hysteroscopic diagnostic pilot study. Acta Cytol 38: 319-323, 1994.

27. Foulks MJ: The Papanicolaou smear: its impact on the promotion of women's health. J Obstet Gynecol Neonatal Nurs 27: 367-373, 1998. 